\title{
Multi-parametric assessment of the anti-angiogenic effects of liposomal glucocorticoids
}

\author{
Ewelina Kluza • Marieke Heisen • Sophie Schmid • Daisy W. J. van der Schaft • \\ Raymond M. Schiffelers • Gert Storm • Bart M. ter Haar Romeny • \\ Gustav J. Strijkers • Klaas Nicolay
}

Received: 9 December 2010/Accepted: 27 December 2010/Published online: 12 January 2011

(C) The Author(s) 2011. This article is published with open access at Springerlink.com

\begin{abstract}
Inflammation plays a prominent role in tumor growth. Anti-inflammatory drugs have therefore been proposed as anti-cancer therapeutics. In this study, we determined the anti-angiogenic activity of a single dose of liposomal prednisolone phosphate (PLP-L), by monitoring tumor vascular function and viability over a period of one week. C57BL/6 mice were inoculated subcutaneously with B16F10 melanoma cells. Six animals were PLP-L-treated and six served as control. Tumor tissue and vascular function were probed using MRI before and at three
\end{abstract}

Ewelina Kluza and Marieke Heisen contributed equally to this study.

Electronic supplementary material The online version of this article (doi:10.1007/s10456-010-9198-5) contains supplementary material, which is available to authorized users.

E. Kluza $(\varangle)$ - S. Schmid · G. J. Strijkers · K. Nicolay Department of Biomedical Engineering, Biomedical NMR, Eindhoven University of Technology, 2.03b N-laag, PO Box 513, 5600 MB Eindhoven, The Netherlands e-mail: E.Kluza@tue.nl

M. Heisen · B. M. ter Haar Romeny

Department of Biomedical Engineering, Biomedical Image Analysis, Eindhoven University of Technology, PO Box 513, 5600 MB Eindhoven, The Netherlands

D. W. J. van der Schaft

Department of Biomedical Engineering, Soft Tissue

Biomechanics and Engineering, Eindhoven University of Technology, PO Box 513, 5600 MB Eindhoven,

The Netherlands

R. M. Schiffelers · G. Storm

Utrecht Institute for Pharmaceutical Sciences (UIPS),

Department of Pharmaceutics, Utrecht University,

PO Box 80.082, 3508 TB Utrecht, The Netherlands timepoints after treatment. DCE-MRI was used to determine $K^{\text {trans }}, v_{\mathrm{e}}$, time-to-peak, initial slope and the fraction of non-enhancing pixels, complemented with immunohistochemistry. The apparent diffusion coefficient (ADC), $\mathrm{T}_{2}$ and tumor size were assessed with MRI as well. PLP-L treatment resulted in smaller tumors and caused a significant drop in $\mathrm{K}^{\text {trans }} 48 \mathrm{~h}$ post-treatment, which was maintained until one week after drug administration. However, this effect was not sufficient to significantly distinguish treated from non-treated animals. The therapy did not affect tumor tissue viability but did prevent the ADC decrease observed in the control group. No evidence for PLP-L-induced tumor vessel normalization was found on histology. Treatment with PLP-L altered tumor vascular function. This effect did not fully explain the tumor growth inhibition, suggesting a broader spectrum of PLP-L activities.

Keywords Anti-angiogenic effects - Tumor-associated inflammation · Glucocorticoids · Liposomes · DCE-MRI

\section{Introduction}

Inflammation is a common feature of the tumor microenvironment, which, according to recent findings, plays a prominent role in tumor growth propagation $[1,2]$. The close relationship between cancer and inflammatory processes prompted the idea of silencing tumor-associated inflammation for therapeutic purposes [3]. Consequently, anti-inflammatory drugs, widely used in clinical practice, have been proposed as potential anti-cancer therapeutics. One of these agents, celecoxib, has recently entered clinical trials, promising a valuable contribution of anti-inflammatory therapy to cancer prevention and treatment [4-7]. 
The anti-tumor activity of glucocorticoids (GC), known as steroid anti-inflammatory agents, has been demonstrated in mouse tumor models [8, 9]. However, adverse effects caused by high daily doses, necessary to achieve tumor growth inhibition, were found to be a major limitation. Recently, encapsulation into long-circulating liposomes was demonstrated to be an effective strategy to enhance intratumoral GC concentration [10] as a result of favorable pharmacokinetic properties of liposomes [11, 12] and the enhanced permeability and retention of tumor tissue [13]. Importantly, the employment of the liposomal drug delivery system dramatically decreased the therapeutic dose and the dosing frequency compared to that of the free drug. For prednisolone phosphate (PLP), which was found to have the most favorable therapeutic index among investigated liposome-encapsulated GC [14], a single intravenous injection of $20 \mathrm{mg}$ PLP/kg/week was sufficient to significantly inhibit tumor growth in B16F10 melanoma and C26 colon carcinoma [10].

Inhibition of angiogenesis was proposed to be a key effect of liposomal GC therapy, leading to tumor growth delay [10]. Findings that support this hypothesis include a decreased expression of multiple pro-angiogenic factors [15] and the inhibition of macrophage activity in the tumor [16], both of which play an important role in the process of neovascularisation. In addition, anti-proliferative effects of liposomal GC on endothelial cells were observed in vitro $[14,15]$. However, the influence of liposomal GC on the structure and the function of the tumor vasculature has not yet been investigated. The evaluation of the tumor vascular response is essential to fully characterize the anti-angiogenic activity of liposomal GC and to provide better understanding of the anti-tumor mechanism.

In the present study, we therefore aimed to determine the anti-angiogenic potency of liposomal prednisolone phosphate (PLP-L) by studying its effect on the tumor vasculature. The efficiency of a single dose of PLP-L (20 mg PLP/kg) was investigated in B16F10 murine melanoma during one week. For comprehensive characterization of the vascular status, both in vivo magnetic resonance imaging (MRI) and histological analyses were performed.

In vivo evaluation of the vascular changes induced by PLP-L was done using dynamic contrast-enhanced MRI (DCE-MRI). This method enables non-invasive characterization of the vessel function, based on the pharmacokinetics of a low molecular weight gadolinium chelate [17]. Due to the sensitivity to changes in vascular density and permeability, DCE-MRI-derived parameters, such as the endothelial transfer constant $\mathrm{K}^{\text {trans }}$, serve as valuable in vivo markers of anti-angiogenic effects [18].

Furthermore, we investigated the effect of PLP-L on the tumor viability, since the expected vascular suppression, by limiting the availability of oxygen and nutrients to rapidly multiplying tumor cells, may lead to cell death. For characterization of this tissue property, we used two DCE-MRIderived parameters, i.e., the fraction of extravascular extracellular space $\left(\mathrm{v}_{\mathrm{e}}\right)$ and the fraction of non-enhancing pixels. Moreover, we assessed the apparent diffusion coefficient (ADC) and the transverse relaxation time $\left(\mathrm{T}_{2}\right)$, which are significant complementary MR indicators of changes in the cellular density and the occurrence of local hemorrhages [19, 20].

As a final step of the vascular response assessment, we performed immunohistochemical examination of tumor tissues after the in vivo measurements, at the end of the study. Two aspects related to the anti-angiogenic activity of PLP-L were investigated. First, we determined the microvessel density (MVD), which is the most established histological marker of the vascular status and response to anti-angiogenic therapy $[21,22]$. Secondly, we investigated whether PLP-L treatment induced tumor vessel normalization, an effect previously described for anti-angiogenic agents [23]. As a marker of vessel maturation we considered the presence of a smooth muscle layer around the vascular endothelium.

\section{Materials and methods}

\section{Preparation and characterization of PLP-L}

PLP-L were prepared as described previously [24]. In short, appropriate amounts of 1,2-dipalmitoyl-sn-glycero3-phosphocholine (DPPC), cholesterol, and 1,2-distearoylsn-glycero-3-phosphoethanolamine-N-[methoxy(polyethylene glycol)-2000] (PEG2000-DSPE), obtained from Avanti Polar Lipids (Albaster, AL, USA), were mixed at 1.85/1/0.15 molar ratio. Subsequently, the lipids were dissolved in chloroform/methanol 1:1 (v/v) and dried by rotary evaporation. The dry lipid film was hydrated in an aqueous solution of $100 \mathrm{mg} / \mathrm{mL}$ of prednisolone phosphate (PLP) (Fagron, Nieuwerkerk a/d IJssel, The Netherlands). The resulting multilamellar lipid vesicles were extruded (Lipofast Extruder, Avestin, Toronto, Canada) 6 times through polycarbonate membrane filters with a pore diameter of 200 and $100 \mathrm{~nm}$ (Costar, Cambridge, MA, USA) at $55^{\circ} \mathrm{C}$. Non-encapsulated PLP was removed using dialysis in HEPES buffered saline ( $\mathrm{pH}$ 7.4) (SigmaAldrich, St. Louis, MO, USA). A dialysis membrane with a molecular weight cutoff between 12 and $14 \mathrm{kDa}$ (Spectrum Laboratories Inc., CA, USA) was used. During $24 \mathrm{~h}$ of dialysis the buffer was repeatedly replenished. The final PLP-L preparation was stored at $4^{\circ} \mathrm{C}$.

The mean diameter and size distribution of the liposomes were determined with dynamic light scattering (Zetasizer Nano, Malvern, UK) at $25^{\circ} \mathrm{C}$. The phospholipid 
content in the liposome suspensions was assessed using phosphate analysis according to Rouser [25] and the concentration of liposome-encapsulated prednisolone phosphate was analyzed with high performance liquid chromatography [15]. A mean diameter of $100 \mathrm{~nm}$ and a narrow size distribution (polydispersity index $=0.1$ ) were determined for the prepared liposomes. The drug and the lipid concentrations were $5 \mathrm{mg} / \mathrm{mL}$ and $60 \mu \mathrm{mol} / \mathrm{mL}$, respectively.

Murine tumor model

B16F10 murine melanoma cells were cultured as a monolayer at $37^{\circ} \mathrm{C}$ and $5 \% \mathrm{CO}_{2}$, in DMEM medium ( 1 g glucose/ L) (Invitrogen, Breda, The Netherlands), supplemented with $10 \%$ fetal bovine serum (Greiner Bio-One, Alphen a/d Rijn, The Netherlands), $2 \mathrm{mmol} / \mathrm{L}$ L-glutamine (Lonza Bioscience, Basel, Switzerland), and $50 \mathrm{U} / \mathrm{mL}$ penicillin/ streptomycin (Lonza Bioscience). The cell line originates from American Type Culture Collection (CRL-6475).

Six to eight week-old C57BL/6 mice (Charles River, Maastricht, The Netherlands) were inoculated with $1 \times 10^{6}$ B16F10 cells subcutaneously in the right flank. Between day 7 and 9 after inoculation, the tumors became palpable in all mice. The animal experiments were approved by the Institutional Ethical Review Committee for animal experiments of Maastricht University (The Netherlands).

\section{Study design}

The timeline of the study is presented in Fig. 1a. The pretreatment MRI examination was performed on a day when tumors became palpable (Day 0). After the pre-treatment MRI scans were acquired, the animals received a single dose of either PLP-L (20 mg PLP/kg) ( $\mathrm{n}=6$, PLP-Ltreated group) or a corresponding volume of saline $(n=6$, Control group) intravenously. The post-treatment MRI examinations were performed on Day 2, Day 4 and Day 6. The time frame of our study was chosen in view of the one week-long therapeutic efficacy of PLP-L [10] and the fast growth of B16F10 tumors. After MRI measurements on Day 6, the mice were sacrificed and the dissected tumors were immunohistochemically analyzed.

\section{MRI acquisition}

MRI measurements were performed with a $6.3 \mathrm{~T}$ scanner (Bruker, Biospin, Ettlingen, Germany), using a $3 \mathrm{~cm}$ birdcage coil (Rapid Biomedical, Rimpar, Germany). The mice were anesthetized with isoflurane and placed in a cradle, equipped with a mask for anesthetic gas and a warm water pad. An infusion line filled with contrast agent was (a)

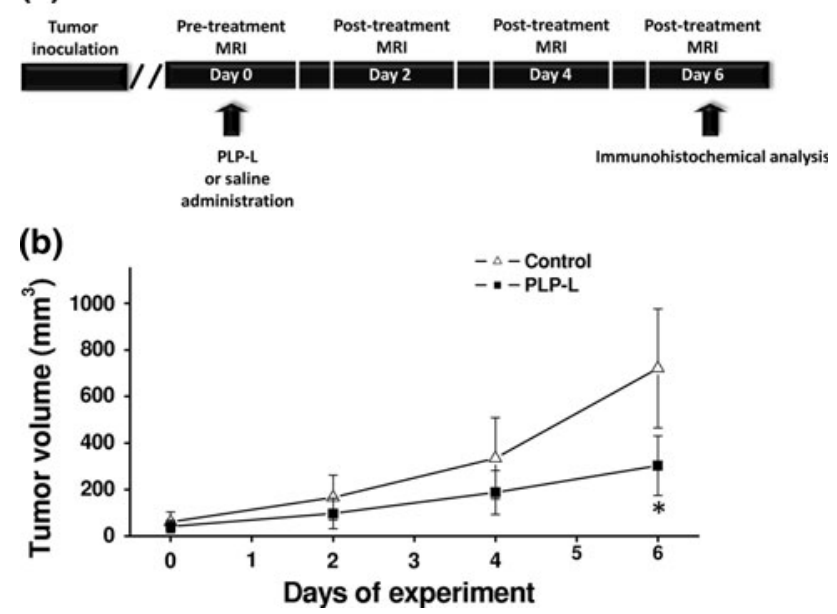

Fig. 1 a Timeline of the study; $\mathbf{b}$ tumor growth curves for PLP-Ltreated and saline-treated mice (Control) (mean $\pm \mathrm{SD}, \mathrm{n}=6)$. "** indicates significant difference in tumor volume between PLP-Ltreated and Control mice on Day 6

placed in the tail vein to enable injection during the MRI experiment. Respiration was monitored with a balloon sensor connected to an ECG/respiratory unit (Rapid Biomedical). The imaging protocol included a fat-suppressed multi-slice $\mathrm{T}_{2}$-weighted spin-echo sequence (TE/TR $=35$ / $4,200 \mathrm{~ms}$, number of averages (NA) $=4$ ) and a multi-slice diffusion-weighted spin-echo sequence $(\mathrm{TE} / \mathrm{TR}=35$ / 2,000 ms, 3 directions, $b$-value $=0,400 \mathrm{~s} / \mathrm{mm}^{2}, \mathrm{NA}=2$ ). Quantitative $T_{2}$ maps were obtained using a multi-echo method, consisting of a series of $\mathrm{T}_{2}$-weighted spin-echo images with 16 different $\mathrm{TE}$ values varying from 9 to $144 \mathrm{~ms}(\mathrm{TR}=2,000 \mathrm{~ms}, \mathrm{NA}=2)$. $\mathrm{T}_{1}$-weighted dynamic contrast-enhanced series (RF-spoiled FLASH, TR/ $\mathrm{TE}=80 / 3.0 \mathrm{~ms}$, flip angle $=50^{\circ}$, number of slices $=8$ ) were acquired for a duration of 25 min with $\mathrm{NA}=2$ $(\mathrm{n}=100$, sampling time $=15.36 \mathrm{~s})$, or NA $=1(\mathrm{n}=200$, sampling time $=7.68 \mathrm{~s}$ ). A dose of $0.3 \mathrm{mmol} / \mathrm{kg}$ of Gadoteridol (Prohance, Bracco Diagnostics, Princeton, NJ, USA) was manually injected as a bolus in the tail vein $80 \mathrm{~s}$ after the start of acquisition. All acquired images had a matrix size of $128 \times 128$, field of view of $3 \times 3 \mathrm{~cm}^{2}$ and $1 \mathrm{~mm}$ slice thickness. The image iso-center and orientation were the same for all scans.

MRI data analysis

Analysis of $T_{2}$ and diffusion-weighted images was performed in Mathematica 6.0 (Wolfram Research Inc., Champaign, IL, USA). Regions of interest (ROIs) were manually defined by drawing contours around the tumor area in every diffusion-weighted image slice in which the tumor was visible. $\mathrm{T}_{2}$-weighted images served as an 
additional reference. Tumor volumes were calculated by multiplying the number of pixels in the tumor ROIs with the pixel volume. ADC maps were generated from fitting diffusion-weighted imaging data according to the equation $\mathrm{ADC}=-1 / \mathrm{b} \ln \left(\mathrm{S} / \mathrm{S}_{0}\right)$, where $\mathrm{S}$ and $\mathrm{S}_{0}$ are signals at $\mathrm{b}=400$ and $\mathrm{b}=0 \mathrm{~s} / \mathrm{mm}^{2}$, respectively [26]. $\mathrm{T}_{2}$ maps were calculated in each slice from mono-exponential decay fitting of the multi-echo data.

DCE-MRI data processing was performed in Matlab (Mathworks, Natick, MA, USA). The analysis included those pixels within the tumor ROI that showed significant signal enhancement, i.e., enhancement equal to or greater than 5 times the noise level. The standard deviation of the noise was determined in air and corrected for the Rician distribution [27]. The fraction of non-enhancing pixels in the total tumor area was quantified and used as a tissue viability-related parameter.

The DCE-MRI signal intensity curves were converted to contrast agent concentration curves $\left(\mathrm{C}_{\mathrm{t}}\right)$ using a previously published reference method [28]. The pre-contrast reference was taken from a user-defined ROI in muscle $(5 \times 5$ voxels). A pre-contrast $T_{1}$ value of $1,285 \mathrm{~ms}$ for muscle [29] and a relaxivity value of $3.7 \mathrm{mM}^{-1} \mathrm{~s}^{-1}$ for Gadoteridol (measured at $6.3 \mathrm{~T}$ at $20^{\circ} \mathrm{C}$ ) were used in the calculations.

The $\mathrm{C}_{\mathrm{t}}$ curves in the selected pixels of the tumor ROI were analyzed. First, by applying the basic two-compartment model by Tofts et al. [30]:

$\mathrm{dC}_{\mathrm{t}} / \mathrm{dt}=\mathrm{K}^{\text {trans }}\left(\mathrm{C}_{\mathrm{p}}-\mathrm{C}_{\mathrm{t}} / \mathrm{v}_{\mathrm{e}}\right)$,

where $\mathrm{v}_{\mathrm{e}}$ is the fraction of extravascular extracellular space (EES), $\mathrm{K}^{\text {trans }}\left(\mathrm{min}^{-1}\right)$ is the volume transfer constant between blood plasma and EES, $\mathrm{C}_{\mathrm{p}}(\mathrm{mM})$ is the contrast agent concentration in the blood plasma space, and $\mathrm{C}_{\mathrm{t}}$ $(\mathrm{mM})$ is the contrast agent concentration in the tissue of interest. The input function $\mathrm{C}_{\mathrm{p}}$ was derived from the contrast agent uptake in skeletal muscle using the reference tissue approach [31, 32], assuming literature values for $\mathrm{K}^{\text {trans }}\left(0.11 \mathrm{~min}^{-1}\right)$ and $\mathrm{v}_{\mathrm{e}}(0.20)$ [31]. To minimize noise propagation, the muscle curve was fitted with an empirical mathematical model [33] prior to this derivation. We used the first $5 \mathrm{~min}$ of the DCE-MRI scan [33]. The fitting was performed with a golden section search method, by minimizing the goodness-of-fit measure $R^{2}$ [34]. In addition to the applied enhancement threshold (enhancement $\geq 5$ times the noise level), we excluded erroneous fits by discarding pixels with a $\mathrm{v}_{\mathrm{e}}$ value equal to or greater than 0.95 .

Secondly, the descriptive parameters time-to-peak $\left(\mathrm{T}_{\text {peak }}\right)$ and initial slope $\left(\mathrm{Slope}_{\mathrm{i}}\right)$ were derived from the $\mathrm{C}_{\mathrm{t}}$ curves [35]. $T_{\text {peak }}(\mathrm{min})$ is the time elapsed between the start of enhancement and the timepoint at which the contrast agent reached its maximal concentration $\left(\mathrm{C}_{\mathrm{t} \text {,peak }}\right)$. Slope $_{\mathrm{i}}(\mathrm{mM} / \mathrm{min})$ approximates the upslope of $\mathrm{C}_{\mathrm{t}}$ :
Slope $_{\mathrm{i}}=\mathrm{C}_{\mathrm{t}, \text { peak }} / \mathrm{T}_{\text {peak }}$.

For consistency, also here only data acquired in the first 5 min was taken into account.

Immunohistochemical analysis of tumor tissues

Tumors were snap-frozen in isopentane and stored at $-80^{\circ} \mathrm{C}$. Five $\mu \mathrm{m}$-thick sections were cut and fixed with icecold acetone for $5 \mathrm{~min}$. In order to assess the microvessel density (MVD), endothelial cells were stained with rat antimouse CD31 primary antibody (BioLegend, San Diego, CA, USA). FITC-conjugated rabbit anti-rat IgG (Dako, Glostrup, Denmark) was used as a secondary antibody. For nuclear staining, 4,6'-diamidino-2-phenylindole (DAPI) (Molecular Probes, Eugene, Oregon, USA) was applied. The tissue samples were examined with a fluorescence microscope (Zeiss, Sliedrecht, The Netherlands). MVD was assessed according to the method described by Weidner et al. [21], by counting the number of vessels in five vascular regions of a section at $200 \times$ magnification. To investigate vessel normalization, another set of tissue sections was stained with monoclonal anti-actin, $\alpha$-smooth muscle-FITC antibody (Sigma-Aldrich). Co-staining was done with rat anti-mouse CD31 antibody (BioLegend) and Alexa Fluor 350-conjugated goat anti-rat IgG (Molecular Probes). The presence of normalized vessels was examined using fluorescence microscope (Zeiss).

Statistical analysis

All data are reported as mean \pm standard deviation (SD). Statistical analysis was performed in SPSS 16.0 (SPSS Inc., Chicago, Illinois, USA). A $t$ test was used to assess the difference between tumor volumes of PLP-L-treated and Control mice at different study timepoints. Statistical analysis of DCE-MRI data was performed on median, 25, 75,95 th percentile values and interquartile range (distance between 25 and 75th percentile values), determined from histograms of $K^{\text {trans }}, v_{e}, T_{\text {peak }}$ and Slope ${ }_{i}$ for each data set [36]. The comparison between the results obtained at different timepoints after drug or saline administration and the baseline readout was done using a paired $t$ test. The comparison of the longitudinal data obtained for both treated and control animals was done using mixed-factors ANOVA for repeated measures, where the within-subject factor was time and the between-subject factor was treatment. For this analysis, we used the relative change in the parameter with respect to its baseline value. The fraction of non-enhancing pixels, $\mathrm{ADC}$ and $\mathrm{T}_{2}$ data were tested using the same statistical methods as described above for the DCE-MRI-derived kinetic parameters. For ADC and $\mathrm{T}_{2}$, the median was used as comparison parameter. The 
analysis of the MVD data was done using a $t$ test. In all analyses, the difference was considered significant at $P<0.05$.

\section{Results}

Effects of PLP-L on tumor growth

To evaluate the effect of PLP-L on tumor growth we monitored lesion size over time, using high resolution MRI measurements. Tumor growth curves, which represent summarized data obtained for the PLP-L-treated and the Control group, are presented in Fig. 1b. During the first days after PLP-L administration (Day 2 and 4), tumor volumes were not significantly affected by the treatment. The growth-inhibiting effect of PLP-L became evident on Day 6, which corresponds to the previous findings [10, 14]. At this timepoint, tumors of PLP-L-treated mice were on average a factor of two smaller than those of the Control group.

Evaluation of anti-angiogenic effects of PLP-L with DCE-MRI

As markers of vascular function, we used $\mathrm{K}^{\text {trans }}, \mathrm{T}_{\text {peak }}$ and Slope $_{i}$, estimated by mathematical modeling of dynamic contrast-enhanced MRI scans. Because $v_{e}$ is a measure of cellular density rather than vessel function, it is discussed in the next section. All assessed parameters are summarized in Table S1 (supplementary material), whereas representative $\mathrm{MR}$ images and $\mathrm{K}^{\text {trans }}$ maps obtained for PLP-L and Control mice are presented in Fig. 2.

Large variability in DCE-MRI parameters was observed already in the pre-treatment measurements (Fig. 3, Table $\mathrm{S} 1$ ), indicating natural inter-tumor differences in vascular function. Therefore, we used baseline measurements as a reference for the assessment of vascular effects. In all PLPL-treated tumors, the first post-treatment examination revealed a reduction in median $\mathrm{K}^{\text {trans }}$ by $27 \pm 14 \%$ compared to the pre-treatment state (Fig. 3a). This significant drop in this parameter was maintained on Day 4 and 6, resulting, on average, in 40 and $45 \%$ decreased median $\mathrm{K}^{\text {trans }}$, respectively, as compared to pre-treatment values. The same trend was observed for the 25 and 75 th $\mathrm{K}^{\text {trans }}$ percentiles (Table S1). For the 95th percentile, which reflects the fraction of $\mathrm{K}^{\text {trans }}$ "hot spots" often associated with intensive tumor growth [37], no change was found at any timepoint (Table S1). Interestingly, for the therapy group the interquartile range was significantly decreased on Day $2(25 \pm 15 \%)$ and $4(33 \pm 25 \%)$, which indicates a more narrow distribution of $\mathrm{K}^{\text {trans }}$ values throughout the enhancing tumor area (Table S1). Moreover, $\mathrm{T}_{\text {peak }}$ was clearly affected by the therapy; after an initial increase of $60 \%$ on Day 2, it was maintained at approximately the same level (Fig. 3c). A significant therapy-induced drop in Slope $_{i}$ was observed only on Day 6. In contrast, in the Control group, no significant change in any of the DCEMRI parameters was observed, neither on Day 2 nor Day 4 (Fig. 3, Table S1). Surprisingly, on the last measurement day (Day 6) a decrease in median $\mathrm{K}^{\text {trans }}(40 \pm 18 \%)$ and
Fig. 2 Representative diffusion-weighted images (DWI), $\mathrm{T}_{1}$-weighted images $\left(\mathrm{T}_{1} \mathrm{w}\right) 60 \mathrm{~s}$ after start of enhancement and corresponding $\mathrm{K}^{\text {trans }}$ maps obtained for a PLP-L-treated and a control mouse before (Day 0) and at different timepoints after PLP-L or saline (Control) administration (Day 2, 4, 6)
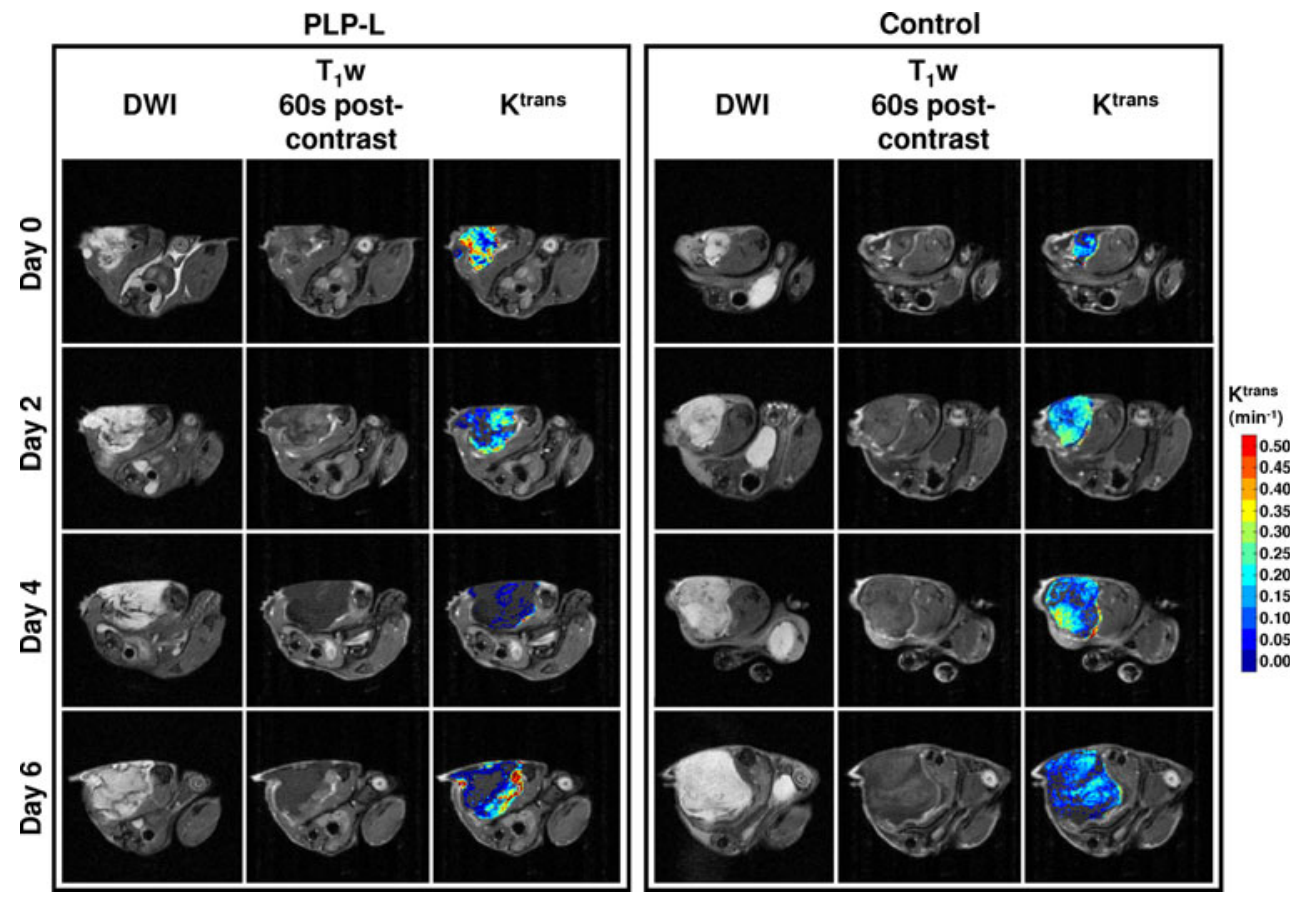
Fig. 3 Influence of PLP-L treatment on median $\mathbf{a} \mathrm{K}^{\text {trans }}$, $\mathbf{b} \mathrm{v}_{\mathrm{e}}, \mathbf{c} \mathrm{T}_{\text {peak }}$ and $\mathbf{d}$ Slope $_{\mathrm{i}}$ in the tumor, compared to the Control (mean $\pm \mathrm{SD}, \mathrm{n}=6$ ). Symbols “*, \#" indicate significant difference compared to baseline (Day 0) for PLP-L (*) and Control group (\#) $(P<0.05)$
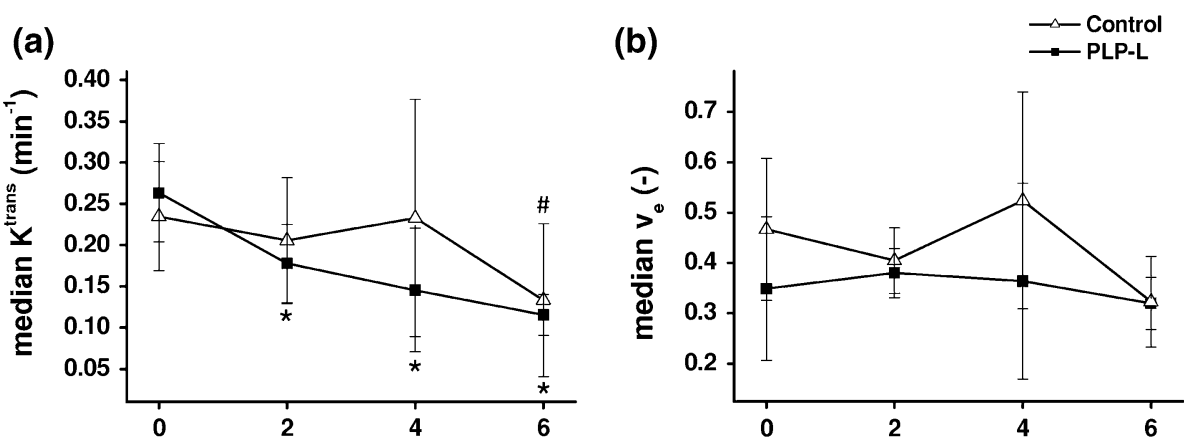

(c)

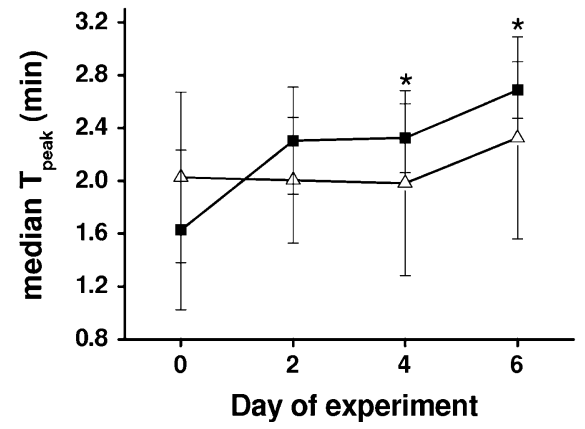

(d)

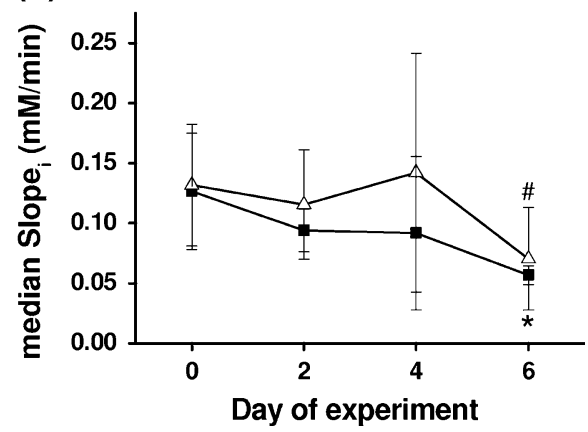

median Slope $\mathrm{i}_{\mathrm{i}}(48 \pm 18 \%)$ became apparent in the Control group (Fig. 3a,d), taking the Day 0 data as a reference.

To test, whether the assessed kinetic parameters could serve as non-invasive imaging markers of the therapeutic response, we performed a statistical comparison between the longitudinal data obtained for the PLP-L-treated and the Control group. The analysis revealed no significant influence of the PLP-L treatment on DCE-MRI-based markers compared to the Control. This result refers to all assessed parameters, indicating that, under the present experimental conditions, DCE-MRI failed to distinguish the response to PLP-L treatment from the normal tumor development.

Evaluation of the tumor viability

The effect of PLP-L treatment on the tumor tissue viability was characterized by means of four MR parameters, $v_{e}$, the fraction of non-enhancing pixels, $\mathrm{ADC}$ and $\mathrm{T}_{2}$. Both DCEMRI-derived markers, i.e., $\mathrm{v}_{\mathrm{e}}$ and the fraction of nonenhancing pixels, did not change significantly after administration of PLP-L (Fig. 3b, Table S1). Similarly, in the Control group they remained stable over time. The results of $A D C$ and $T_{2}$ measurements are summarized in Fig. 4. Neither ADC nor $T_{2}$ were significantly affected by the treatment with PLP-L. The constant median values of ADC and $\mathrm{T}_{2}$ of approximately $0.83 \times 10^{-3} \mathrm{~mm}^{2} / \mathrm{s}$ and $62 \mathrm{~ms}$, respectively, throughout the investigation suggest that the therapy did not cause necrotic changes in the tumor. Interestingly, for the Control group we observed a decrease in median ADC over time (Fig. 4a). A significant drop compared to the starting point was noted already on Day 2. The trend of decreasing ADC observed throughout the experiment suggests that the normal tumor growth is accompanied by an increase in cellular density. On the other hand, $\mathrm{T}_{2}$ remained relatively stable (Fig. $4 \mathrm{~b}$ ). For
Fig. 4 Effect of PLP-L on median (a) apparent diffusion coefficient (ADC) and b transverse relaxation time $\left(\mathrm{T}_{2}\right)$ in the tumor, compared to the Control (mean $\pm \mathrm{SD}, \mathrm{n}=6$ ). Symbol "*" indicates significantly decreased median ADC values compared to baseline measurements reported for the Control (a)

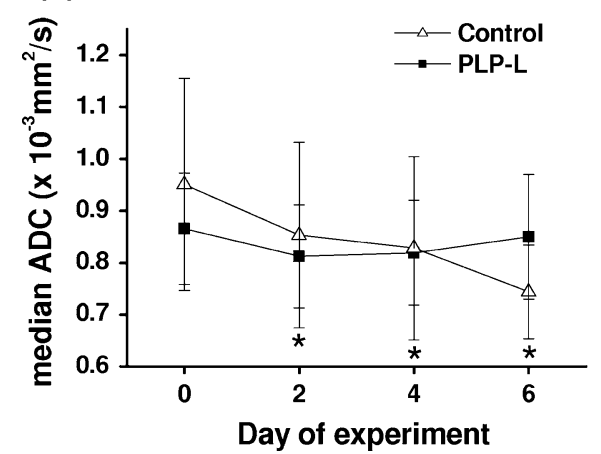

(b)

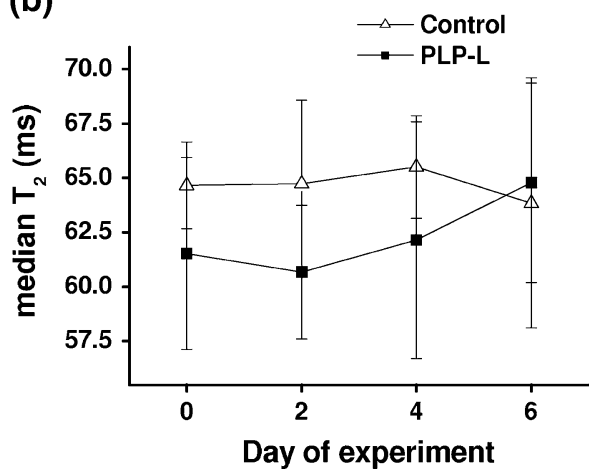


Fig. 5 a Representative fluorescence microscopy images of tumor sections from Control and PLP-L-treated mice. CD31stained endothelial cells are shown in green and cell nuclei in blue (DAPI). b Microvessel density (MVD) assessed for Control (white bar) and PLP-Ltreated (grey bar) tumors. Bars represent mean $\pm \mathrm{SD}(\mathrm{n}=6)$ (a)
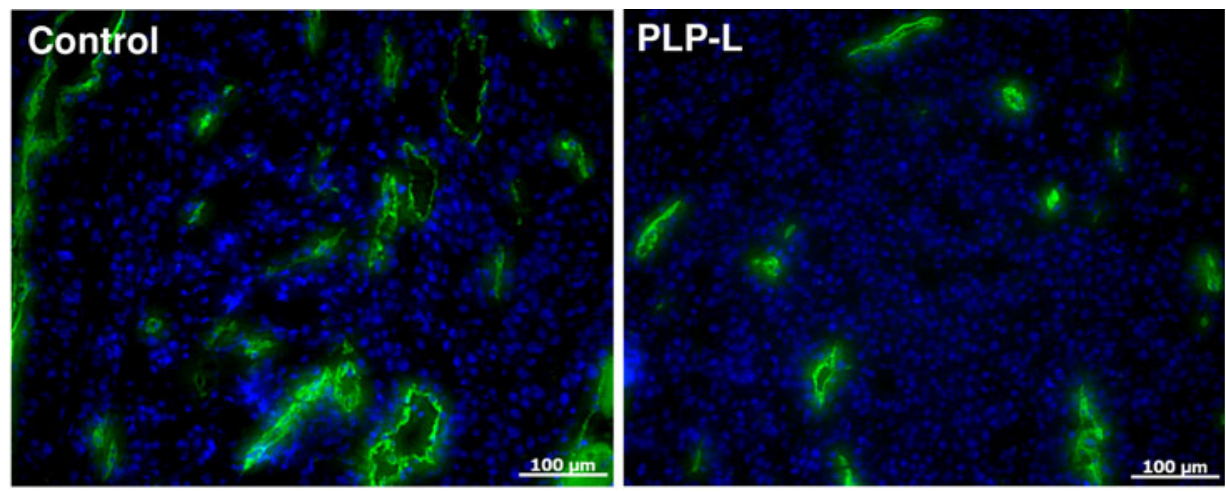

(b)

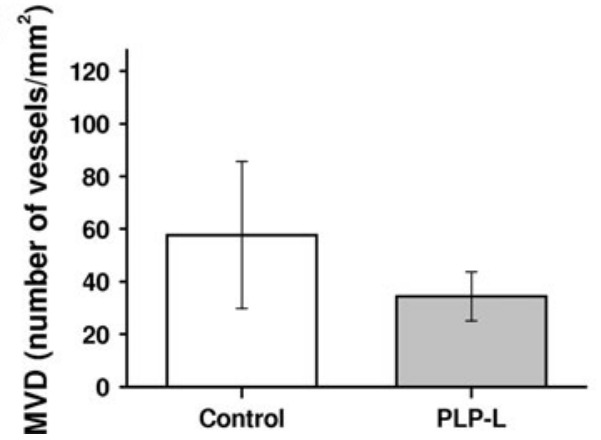

neither of the aforementioned parameters we found a significant influence of the PLP-L treatment compared to the Control.

Microvessel density and vessel normalization

Ex vivo analysis of tumor tissues was performed after MRI experiments, thus, one week after administration of PLP-L or saline. First, we assessed the microvessel density (MVD), which is an important marker of the tumor angiogenic activity. The summarized results of this analysis are presented in Fig. 5. The average MVD of 34 vessels $/ \mathrm{mm}^{2}$ assessed in PLP-L-treated tumors was lower compared to 58 vessels $/ \mathrm{mm}^{2}$ in the Control group. Nevertheless, statistical analysis did not confirm a significant difference in MVD. In addition, we investigated the relation between MVD and $\mathrm{K}^{\text {trans }}$, being important ex vivo and in vivo markers of the vascular status, respectively. However, we did not find a correlation between these two parameters $\left(r^{2}=0.23\right)$.

To investigate vascular maturation, which was considered as a potential effect of PLP-L, the tumor sections were examined for the presence of the smooth muscle layer around the endothelium of tumor vessels. Both PLP-Ltreated and Control tumor sections were analyzed and compared to normal muscle tissue, which was used as a reference. In Fig. 6a, a normal vessel of the muscle is presented. It is characterized by a continuous rim of smooth muscle cells around the endothelium. We did not observe such well-formed vessels in both PLP-L-treated and Control tumors (Fig. 6b,c). Predominantly, tumor vessel walls were composed of a bare endothelial cell layer. If smooth muscle staining was present in the tumor tissue, it appeared to be disturbed and poorly defined (Fig. 6c). This observation refers to both the PLP-L-treated and the Control group, which suggests that this is not a therapy-induced effect.

\section{Discussion}

In the current study, we evaluated the response of tumor vasculature to PLP-L treatment using in vivo MRI and immunohistochemical analyses. DCE-MRI monitoring revealed therapy-induced vascular alterations that were manifested by a significant decrease in $\mathrm{K}^{\text {trans }}$ and concomitant increase in $\mathrm{T}_{\text {peak }}$. At the same time, the treatment did not influence any of the MR parameters related to the tumor tissue viability. Interestingly, PLP-L administration prevented the drop in ADC values over time that was observed in non-treated tumors. Surprisingly, the tumor growth in the Control group was also accompanied by a significant decrease in $\mathrm{K}^{\text {trans }}$ and $\mathrm{Slope}_{\mathrm{i}}$ one week after start of the experiment. Moreover, at this timepoint, neither a statistically important difference in MVD between the PLP-L-treated and the Control group, nor signs of therapyinduced tumor vessel normalization were found. The current findings provide a rather complex picture of the vascular effects of PLP-L. On one hand, the DCE-MRI results support the thesis on angiogenesis inhibition underlying 
Fig. 6 Representative

fluorescence microscopy images of the tumor and surrounding muscle tissue. Anti- $\alpha$-SMA staining of smooth muscle cells is shown in green and CD31 staining of endothelial cells in blue. a An example of a mature vessel in skeletal muscle, showing a ring of smooth muscle cells around endothelium. b The border region between the tumor and the normal muscle tissue, containing anti- $\alpha$-SMA-negative vessels (tumor) and multiple vessels with pronounced anti- $\alpha$ SMA staining (indicated with white arrows in muscle tissue). c Tumor vasculature, among which a single vessel was found to have a discontinuous smooth muscle layer (indicated with white arrow and shown in a higher magnification in the inset)
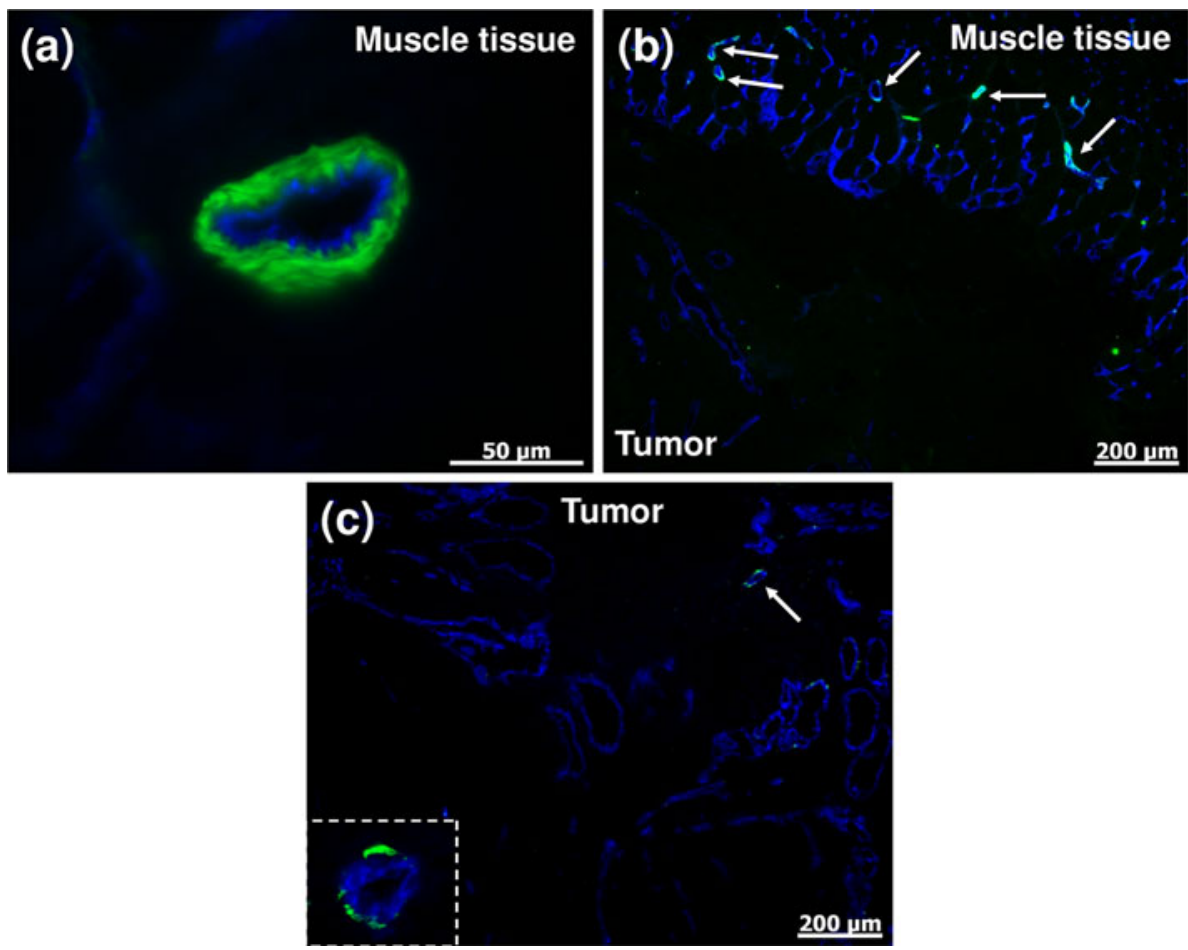

tumor growth suppression by PLP-L. However, we did not find a significant difference in vascular parameters between PLP-L-treated and Control tumors, which we attribute to high inter-tumor variability and vascular alterations that accompany normal tumor development.

Glucocorticoids (GC) are among the most effective anti-inflammatory and immunosuppressive agents [38]. In clinical oncology, they are included in all therapeutic regimes of lymphatic tissue malignancies [39]. Moreover, they are used as a part of endocrine therapy in breast and prostate cancer, and as anti-edema agents in brain tumors $[39,40]$. GC are also widely applied in prevention and treatment of chemotherapy-associated side effects, such as nausea and allergic reactions. However, the influence of $\mathrm{GC}$ on the growth of solid tumors is a topic of ongoing scientific debate. This is due to the unclear role of the glucocorticoid receptor in the regulation of malignant cells and the still controversial idea of antiimmune therapy as anti-cancer strategy [39, 40]. Nevertheless, the broad spectrum of GC activities, including anti-inflammatory, anti-angiogenic, anti-edema, necrotic and apoptotic actions, appears to be very attractive for the treatment of such a complex disease as cancer. In support of this, several pre-clinical investigations demonstrated the therapeutic efficacy of GC in animal tumor models $[8,9]$. In contrast, other pre-clinical and, to some extent, clinical data suggest that GC induce resistance to chemo- and radio-therapy and increase metastatic potential [40].
In this study, we confirmed the previously reported inhibitory effect of PLP-L on the growth of B16F10 melanoma. The mechanism underlying this effect may involve the complex interaction of the drug with the tumor microenvironment. Therefore, versatile techniques, such as MRI, which provide extensive morphological and functional information, are especially attractive for therapy evaluation. Moreover, the application of non-invasive methods facilitates translation from the preclinical to the clinical setting. MR measurements of water diffusion and vessel function were previously employed to study the effects of GC therapy in brain tumors. Several patient studies demonstrated a significantly reduced mean diffusivity in peritumoral edematous brain and ADC in the tumor after dexamethasone treatment [41, 42]. Moreover, GC were found to decrease blood-tumor barrier permeability. Armitage et al. [43] integrated diffusion, perfusion and permeability MRI to characterize the response of intracranial tumors to dexamethasone. Significantly reduced $\mathrm{K}^{\text {trans }}, \mathrm{v}_{\mathrm{e}}$, and $\mathrm{v}_{\mathrm{p}}$ (the fractional plasma volume) were observed $48-72 \mathrm{~h}$ after treatment in glioblastoma, however, not in meningioma and metastatic carcinoma. Furthermore, Crokart et al. [44] performed DCE-MRI using the rapid-clearance blood pool agent P792 (Vistarem) to assess tumor perfusion and permeability $30 \mathrm{~min}$ after hydrocortisone administration. The fraction of perfused pixels of $37.7 \pm 10.8 \%$ in treated tumors was found to be significantly lower than in Control tumors. However, neither $K^{\text {trans }}, v_{p}$ nor the redistribution rate constant $k_{e p}$ 
were affected. All the aforementioned studies have shown the potential of MRI for detection of the anti-tumor effects of steroid anti-inflammatory agents.

To monitor the response of tumor vasculature to therapy with PLP-L, we used DCE-MRI, which is currently the leading in vivo method for the assessment of antiangiogenic effects [18, 45]. Generally, we observed a large variability between individuals, starting already at the pre-treatment state. By using baseline measurements as a reference, we found significantly reduced median $\mathrm{K}^{\text {trans }}$ after the PLP-L treatment. Moreover, a decreased interquartile range of $\mathrm{K}^{\text {trans }}$ distribution on Day 2 and 4 indicated a more homogenous tumor vascular function in PLP-L-treated mice. The suppressive effect on tumor vasculature was further supported by significantly increased $\mathrm{T}_{\text {peak }}$. However, when the summarized data of the PLP-L-treated and the Control mice were compared, no clear anti-angiogenic effect of PLP-L was detected. We believe that this is due to the aforementioned intertumor variability of vessel function, observed throughout the course of the experiment. Moreover, we found that non-treated tumors followed eventually the same trend of changes in vascular parameters as PLP-treated lesions. This occurred later in time, on Day 6, indicating tumor growth-dependent factors as a source of the decreased vascular function in the Control group. We avoided the interference of necrotic regions with these results, as nonenhancing pixels were excluded from DCE-MRI analysis. However, another important tumor tissue property, the interstitial fluid pressure, which is known to increase with tumor growth [46], could have played a role in the observed effect. Interestingly, on Day 6, we also did not find a significant difference in MVD between the PLP-Ltreated and the Control group. At the same time, MVD did not correlate with median $\mathrm{K}^{\text {trans }}$ assessed on Day 6 . The dissociation between MRI-based and histopathological parameters can be explained by the different character of these measures [37]. Despite that, both DCE-MRI and histological readout led to the same conclusion that PLP-L did not affect the vasculature significantly compared to the Control.

The extraction of separate estimates of blood flow and vascular permeability, as well as vascular fraction $\left(\mathrm{v}_{\mathrm{p}}\right)$, by using more complex pharmacokinetics modeling, might provide additional insights into the vascular effects of PLPL [47]. In the present study, we applied the basic Tofts model, which does not incorporate the vascular fraction $\left(\mathrm{v}_{\mathrm{p}}\right)$ as a model parameter. This was motivated by too low temporal resolution of the $\mathrm{T}_{1}$-weighted dynamic scan. According to Henderson et al. [48], reliable $\mathrm{v}_{\mathrm{p}}$ assessment requires a temporal resolution of at least $4 \mathrm{~s}$ for human data. Separate estimates of flow and permeability instead of $\mathrm{K}^{\mathrm{trans}}$ require even higher temporal resolution [49].
As supportive parameters we used those related to the tumor tissue viability, i.e., $v_{\mathrm{e}}$, the fraction of non-enhancing pixels, ADC and $\mathrm{T}_{2}$. We found that the PLP-L treatment did not result in significant changes in either of the aforementioned parameters, indicating undisturbed tumorcell density and stable necrotic fraction. Interestingly, we reported a consistent decay of the tumor ADC over time in the Control group. Since this was not observed after PLP-L administration, we can conclude that the treatment does not only affect the tumor size but also prevents an increase of tumor-cell density.

An important point of our investigation was to determine, whether angiogenesis-related parameters are suitable markers of the therapeutic efficacy of PLP-L. Previous studies on the anti-angiogenic effects of PLP-L were focused predominantly on molecular markers of angiogenesis. Banciu et al. [15] reported a significantly reduced expression of the basic fibroblast growth factor and a wide range of inflammatory mediators involved in the process of angiogenesis. At the same time, the vascular endothelial growth factor was not affected by the treatment. We hypothesized that the diagnostic criteria used for angiogenesis inhibitors can be also applied for PLP-L. However, we found that neither MR nor histopathological vascular markers were capable of differentiating between the PLPL-treated and the Control tumors. This was surprising, since the PLP-L treatment resulted in an over $40 \%$ decrease in median $\mathrm{K}^{\text {trans }}$, which is often considered a true positive response to the anti-angiogenic treatment [18]. However, this was also observed for the Control group on Day 6 . Therefore, the assessed vascular parameters appear to be of limited value as biomarkers of the response to liposomal GC. Ideally, the biological endpoint should relate specifically to the mechanism of the therapeutic action. However, due to a broad spectrum of GC activities, the identification of a specific therapeutic marker might be very challenging. Considering the previous findings [15, 16], the most promising markers appear to be those related to the silencing effects of GC on the tumor-associated inflammation. The non-invasive assessment of this type of information requires the use of cellular and molecular imaging techniques.

In conclusion, we provided evidence that treatment with PLP-L suppresses the functioning of tumor vasculature, which can subsequently lead to tumor growth inhibition. The anti-angiogenic effects of PLP-L were manifested by a significant drop in tumor $\mathrm{K}^{\text {trans }} 48 \mathrm{~h}$ post-treatment, which was maintained until one week after drug administration. However, we also observed naturally occurring vascular alterations in the Control group. The obtained results suggest that angiogenesis monitoring does not provide sufficient information on the therapeutic efficacy of PLP-L. Therefore, more specific markers of the anti-tumor activity 
of liposomal glucocorticoids are desired. Among the potential candidates are those directly related to the suppression of inflammatory processes in the tumor.

Acknowledgments This study was partly funded by the European Network of Excellence Diagnostic Molecular Imaging (DIMI, LSHBCT-2005-512146), and the Integrated European Union Project Targeted Delivery of Nanomedicine (MEDITRANS, FP6-2004-NMP-NI4/IP 026668-2). This study was performed in the framework of the European Cooperation in Science and Technology (COST) D38 Action Metal-Based Systems for Molecular Imaging Applications. We thank Louis van Bloois from Utrecht University for HPLC measurements.

Open Access This article is distributed under the terms of the Creative Commons Attribution Noncommercial License which permits any noncommercial use, distribution, and reproduction in any medium, provided the original author(s) and source are credited.

\section{References}

1. Coussens LM, Werb Z (2002) Inflammation and cancer. Nature 420(6917):860-867. doi:10.1038/nature01322

2. Mantovani A, Allavena P, Sica A, Balkwill F (2008) Cancerrelated inflammation. Nature 454(7203):436-444. doi:10.1038/ nature 07205

3. Schiffelers RM, de Visser KE (2009) Inflammation as target for pharmaceutical intervention in cancer. Curr Pharm Des 15(16):1822-1824

4. Mantovani G, Macciò A, Madeddu C, Serpe R, Antoni G, Massa E et al (2010) Phase II nonrandomized study of the efficacy and safety of COX-2 inhibitor celecoxib on patients with cancer cachexia. J Mol Med 88(1):85-92. doi:10.1007/s00109-0090547-z

5. Debucquoy A, Roels S, Goethals L, Libbrecht L, Cutsem EV, Geboes K et al (2009) Double blind randomized phase II study with radiation +5 -fluorouracil \pm celecoxib for resectable rectal cancer. Radiother Oncol 93(2):273-278. doi:10.1016/j.radonc. 2009.08.006

6. Bhatt RS, Merchan J, Parker R, Wu HK, Zhang L, Seery V et al (2010) A phase 2 pilot trial of low-dose, continuous infusion, or "metronomic" paclitaxel and oral celecoxib in patients with metastatic melanoma. Cancer 116(7):1751-1756. doi:10.1002/ cncr. 24902

7. Sooriakumaran P, Coley HM, Fox SB, Macanas-Pirard P, Lovell DP, Henderson A et al (2009) A randomized controlled trial investigating the effects of celecoxib in patients with localized prostate cancer. Anticancer Res 29(5):1483-1488

8. Folkman J, Langer R, Linhardt RJ, Haudenschild C, Taylor S (1983) Angiogenesis inhibition and tumor regression caused by heparin or a heparin fragment in the presence of cortisone. Science 221(4612):719-725. doi:10.1126/science.6192498

9. Penhaligon M, Camplejohn RS (1985) Combination heparin plus cortisone treatment of two transplanted tumors in $\mathrm{C} 3 \mathrm{H} / \mathrm{He}$ mice. J Natl Cancer Inst 74(4):869-873

10. Schiffelers RM, Metselaar JM, Fens MHAM, Janssen APCA, Molema G, Storm G (2005) Liposome-encapsulated prednisolone phosphate inhibits growth of established tumors in mice. Neoplasia 7(2):118-127. doi:10.1593/neo.04340

11. Drummond DC, Meyer O, Hong K, Kirpotin DB, Papahadjopoulos D (1999) Optimizing liposomes for delivery of chemotherapeutic agents to solid tumors. Pharmacol Rev 51(4):691-743
12. Drummond DC, Noble CO, Hayes ME, Park JW, Kirpotin DB (2008) Pharmacokinetics and in vivo drug release rates in liposomal nanocarrier development. J Pharm Sci 97(11):4696-4740. doi:10.1002/jps. 21358

13. Greish K (2010) Enhanced permeability and retention (EPR) effect for anticancer nanomedicine drug targeting. Methods Mol Biol 624:25-37

14. Banciu M, Fens MHAM, Storm G, Schiffelers RM (2008) Antitumor activity and tumor localization of liposomal glucocorticoids in B16 melanoma-bearing mice. J Control Release 127(2):131-136. doi:10.1016/j.jconrel.2008.01.008

15. Banciu M, Schiffelers RM, Fens MHAM, Metselaar JM, Storm G (2006) Anti-angiogenic effects of liposomal prednisolone phosphate on B16 melanoma in mice. J Control Release 113(1):1-8. doi:10.1016/j.jconrel.2006.03.019

16. Banciu M, Metselaar JM, Schiffelers RM, Storm G (2008) Antitumor activity of liposomal prednisolone phosphate depends on the presence of functional tumor-associated macrophages in tumor tissue. Neoplasia 10(2):108-117. doi:10.1593/neo.07913

17. Jackson A, O'Connor JPB, Parker GJM, Jayson GC (2007) Imaging tumor vascular heterogeneity and angiogenesis using dynamic contrast-enhanced magnetic resonance imaging. Clin Cancer Res 13(12):3449-3459. doi:10.1158/1078-0432.CCR-070238

18. O'Connor JPB, Jackson A, Parker GJM, Jayson GC (2007) DCEMRI biomarkers in the clinical evaluation of antiangiogenic and vascular disrupting agents. Br J Cancer 96(2):189-195. doi: 10.1038/sj.bjc.6603515

19. Moffat BA, Hall DE, Stojanovska J, McConville PJ, Moody JB, Chenevert TL et al (2004) Diffusion imaging for evaluation of tumor therapies in preclinical animal models. MAGMA 17(3-6):249-259. doi:10.1007/s10334-004-0079-z

20. Carano RAD, Ross AL, Ross J, Williams SP, Koeppen H, Schwall RH et al (2004) Quantification of tumor tissue populations by multispectral analysis. Magn Reson Med 51(3):542-551. doi: $10.1002 / \mathrm{mrm} .10731$

21. Weidner N, Semple JP, Welch WR, Folkman J (1991) Tumor angiogenesis and metastasis-correlation in invasive breast carcinoma. N Engl J Med 324(1):1-8

22. Bertolini F, Mancuso P, Shaked Y, Kerbel RS (2007) Molecular and cellular biomarkers for angiogenesis in clinical oncology. Drug Discov Today 12(19-20):806-812. doi:10.1016/j.drudis. 2007.08.011

23. Jain RK (2005) Normalization of tumor vasculature: an emerging concept in antiangiogenic therapy. Science 307(5706):58-62. doi: 10.1126/science. 1104819

24. Metselaar JM, Wauben MHM, Wagenaar-Hilbers JPA, Boerman OC, Storm G (2003) Complete remission of experimental arthritis by joint targeting of glucocorticoids with long-circulating liposomes. Arthritis Rheum 48(7):2059-2066. doi:10.1002/art. 11140

25. Rouser G, Fkeischer S, Yamamoto A (1970) Two dimensional thin layer chromatographic separation of polar lipids and determination of phospholipids by phosphorus analysis of spots. Lipids 5(5):494-496

26. Stejskal E, Tanner J (1965) Spin diffusion measurements: spin echoes in the presence of a time-dependent field gradient. J Chem Phys 42(1):288

27. Kaufman L, Kramer DM, Crooks LE, Ortendahl DA (1989) Measuring signal-to-noise ratios in MR imaging. Radiology 173(1):265-267

28. Medved M, Karczmar G, Yang C, Dignam J, Gajewski TF, Kindler $\mathrm{H}$ et al (2004) Semiquantitative analysis of dynamic contrast enhanced MRI in cancer patients: variability and changes in tumor tissue over time. J Magn Reson Imag 20(1):122-128. doi:10.1002/jmri.20061 
29. Marzola P, Mocchegiani E, Nicolato E, Tibaldi A, Sbarbati A, Osculati F (1999) Chemical shift imaging at 4.7 tesla of thymus in young and old mice. J Magn Reson Imag 10(1):97-101

30. Tofts PS, Brix G, Buckley DL, Evelhoch JL, Henderson E, Knopp MV et al (1999) Estimating kinetic parameters from dynamic contrast-enhanced T(1)-weighted MRI of a diffusable tracer: standardized quantities and symbols. J Magn Reson Imag 10(3):223-232

31. Kovar DA, Lewis M, Karczmar GS (1998) A new method for imaging perfusion and contrast extraction fraction: input functions derived from reference tissues. J Magn Reson Imag 8(5):1126-1134

32. Heisen M, Fan X, Buurman J, van Riel NAW, Karczmar GS, ter Haar Romeny BM (2010) The use of a reference tissue arterial input function with low temporal resolution DCE-MRI data. Phys Med Biol 55(16):4871-4883. doi:10.1088/0031-9155/55/16/016

33. Fan X, Medved M, River JN, Zamora M, Corot C, Robert P et al (2004) New model for analysis of dynamic contrast-enhanced MRI data distinguishes metastatic from nonmetastatic transplanted rodent prostate tumors. Magn Reson Med 51(3):487-494. doi: $10.1002 / \mathrm{mrm} .10737$

34. Press WH, Teukolsky SA, Vetterling WT, Flannery BP (1996) Numerical recipes in Fortran 77: the art of scientific computing, 2nd edn. Cambridge University Press, New York

35. Szabó BK, Aspelin P, Wiberg MK, Boné B (2003) Dynamic MR imaging of the breast. Analysis of kinetic and morphologic diagnostic criteria. Acta Radiol 44(4):379-386

36. Furman-Haran E, Schechtman E, Kelcz F, Kirshenbaum K, Degani H (2005) Magnetic resonance imaging reveals functional diversity of the vasculature in benign and malignant breast lesions. Cancer 104(4):708-718. doi:10.1002/cncr.21225

37. de Lussanet QG, Backes WH, Griffioen AW, Padhani AR, Baeten CI, van Baardwijk A et al (2005) Dynamic contrast-enhanced magnetic resonance imaging of radiation therapy-induced microcirculation changes in rectal cancer. Int J Radiat Oncol Biol Phys 63(5):1309-1315. doi:10.1016/j.ijrobp.2005.04.052

38. Franchimont D (2004) Overview of the actions of glucocorticoids on the immune response: a good model to characterize new pathways of immunosuppression for new treatment strategies. Ann N Y Acad Sci 1024:124-137. doi:10.1196/annals.1321.009

39. Coleman RE (1992) Glucocorticoids in cancer therapy. Biotherapy $4(1): 37-44$

40. Herr I, Pfitzenmaier J (2006) Glucocorticoid use in prostate cancer and other solid tumours: implications for effectiveness of cytotoxic treatment and metastases. Lancet Oncol 7(5):425-430. doi:10.1016/S1470-2045(06)70694-5

41. Bastin ME, Delgado M, Whittle IR, Cannon J, Wardlaw JM (1999) The use of diffusion tensor imaging in quantifying the effect of dexamethasone on brain tumours. Neuroreport 10(7): $1385-1391$

42. Minamikawa S, Kono K, Nakayama K, Yokote H, Tashiro T, Nishio A et al (2004) Glucocorticoid treatment of brain tumor patients: changes of apparent diffusion coefficient values measured by MR diffusion imaging. Neuroradiology 46(10):805-811. doi:10.1007/s00234-004-1268-1

43. Armitage PA, Schwindack C, Bastin ME, Whittle IR (2007) Quantitative assessment of intracranial tumor response to dexamethasone using diffusion, perfusion and permeability magnetic resonance imaging. Magn Reson Imag 25(3):303-310. doi: 10.1016/j.mri.2006.09.002

44. Crokart N, Jordan BF, Baudelet C, Cron GO, Hotton J, Radermacher K et al. (2007) Glucocorticoids modulate tumor radiation response through a decrease in tumor oxygen consumption. Clin Cancer Res 13(2 Pt 1):630-635. doi:10.1158/1078-0432. CCR-06-0802

45. Leach MO, Brindle KM, Evelhoch JL, Griffiths JR, Horsman MR, Jackson A et al (2005) The assessment of antiangiogenic and antivascular therapies in early-stage clinical trials using magnetic resonance imaging: issues and recommendations. Br J Cancer 92(9):1599-1610. doi:10.1038/sj.bjc.6602550

46. Sevick EM, Jain RK (1989) Geometric resistance to blood flow in solid tumors perfused ex vivo: effects of tumor size and perfusion pressure. Cancer Res 49(13):3506-3512

47. Donaldson SB, West CML, Davidson SE, Carrington BM, Hutchison G, Jones AP, Sourbron SP, Buckley DL (2010) A comparison of tracer kinetic models for T1-weighted dynamic contrast-enhanced MRI: application in carcinoma of the cervix. Magn Reson Med 63(3):691-700. doi:10.1002/mrm.22217

48. Henderson E, Rutt BK, Lee TY (1998) Temporal sampling requirements for the tracer kinetics modeling of breast disease. Magn Reson Imag 16(9):1057-1073. doi:10.1016/S0730-725X (98)00130-1

49. Henderson E, Sykes J, Drost D, Weinmann HJ, Rutt BK, Lee TY (2000) Simultaneous MRI measurement of blood flow, blood volume, and capillary permeability in mammary tumors using two different contrast agents. J Magn Reson Imag 12(6): 991-1003 